\title{
Living Alone is Associated with Poorer Muscular Strength, Lower 25-hydroxy-vitamin D and Lower Bone Mineral Density in Icelandic Community Dwelling Old Adults
}

\section{Geirsdottir OG ${ }^{1}$, Jonsson PV ${ }^{2,3}$, Thorsdottir $\mathrm{I}^{4}$ and Ramel $\mathrm{A}^{1 *}$}

${ }^{1}$ Faculty of Food Science and Nutrition, University of Iceland, Reykjavik, Iceland

${ }^{2}$ The Icelandic Gerontological Research Center, Reykjavik, Iceland

${ }^{3}$ Department of Geriatrics, National University Hospital of Iceland, Reykjavik,

Iceland

${ }^{4}$ School of Health Sciences, University of Iceland, Reykjavik, Iceland

*Corresponding Author: Ramel A, Professor, Faculty of Food Science and Nutrition,

University of Iceland, Iceland.
Received: June 16, 2021

Published: September 16, 2021

(C) All rights are reserved by Ramel A., et al.

\begin{abstract}
Background: Living alone has been a significant public health concern among older adults given its association with a wide range of adverse health outcomes. The aim of this study was to examine whether living alone is associated with physical strength and bone health in community-dwelling older adults.

Methods: This was a secondary analysis of existing cross-sectional data of old adults $(\mathrm{N}=182,73.7 \pm 5.7 \mathrm{yrs}, 58.2 \%$ female) from the Reykjavik capital area in Iceland. Information on socioeconomics, health, dietary intake and physical function was collected. 25-hydroxy-vitamin D (250HD) and bone mineral density (BMD) were measured. Participants were grouped retrospectively into "living alone" and into "in cohabitation".

Results: Of our subjects, $76.4 \%$ were in cohabitation and and $23.6 \%$ lived alone. Participants who lived alone were older $(74.5$ \pm 5.6 vs. $72.1 \pm 5.0, \mathrm{P}=0.008)$ and more often female (74.4 vs. $53.2 \%, \mathrm{P}=0.014)$, but there were no differences in education, smoking, number of medications, physical activity (PA) or body mass index (BMI). According to age and gender corrected analyses, participants in cohabitation had higher grip strength $(6.2 \pm 2.4 \mathrm{lb}, \mathrm{P}=0.011)$, higher $250 \mathrm{HD}(13.1 \pm 6.3 \mathrm{nmol} / \mathrm{L}, \mathrm{P}=0.037)$ and higher BMD (z-score lumbal: $1.195 \pm 0.417, \mathrm{P}=0.005$; z-score femur: $0.421 \pm 0.219, \mathrm{P}=0.054$; z-score total: $0.846 \pm 0.290, \mathrm{P}=0.004$ ). Statistical correction for PA, BMI, education and fish oil intake did not change the results.

Conclusion: In comparison to old adults who live in cohabitation, Icelandic old adults who live alone have poorer physical strength, lower 250HD and lower BMD, which can increase their risk for wrist- or hip fracture. These differences between groups were not explained by physical, dietary or social confounding variables.
\end{abstract}

Keywords: Osteoporosis; Old Adults; Socioeconomic Status

\section{Introduction}

Osteoporosis, i.e., low bone mass and deterioration of bone tissue, is a serious health issue, particularly as populations in Western countries age rapidly. This condition can lead to an increased risk of bone fractures [1,2], with high costs of treatment creating a financial burden for those involved and the society. Additionally, fractures can seriously decrease physical function and mobility, negatively impact quality of life, and result into an increased mor- 
tality rate $[3,4]$. Appropriate strategies targeting the most vulnerable populations are desperately needed.

In general, social factors such as socioeconomic status, social support, relationship and religion are related to health and consequently there is also some evidence documenting that there are vulnerable groups within a society being linked to low bone mineral density (BMD) and higher fracture risk. E.g., low socioeconomic status (SES) has been reported to be associated with low BMD and higher risk of osteoporosis [5,6]. Loneliness and living alone have been significant public health concerns among older adults given their associations with a wide range of adverse health outcomes. However, the overall evidence is limited regarding the relationship between bone health and living alone vs. living in cohabitation [711].

A study from Korea reported that individuals living alone had significantly lower BMD and greater odds of osteoporosis than individuals living in cohabitation [7]. In earlier published case-control studies living alone was independently associated with increased fracture risk in Norway [8] and in Denmark [9]. Also, living alone was associated with increased fracture risk in a population-based study in Finish women [10]. However, an Australian study provides equivocal evidence reporting that living alone was associated with lower bone mineral content (BMC) in Australian-born men but related to higher femoral neck BMC in immigrants from Southern Europe [11].

Several of the above mentioned studies fall short to investigate the potential mechanisms for the observed differences between social groups. These mechanisms are not fully understood but may involve some known modifiable risks and preventive behavioral factors, including body mass index (BMI), physical exercise, cigarette smoking, alcohol consumption and use of anti-osteoporotic drugs [12-17].

In order to gain knowledge on the relationship between living alone, bone health and lifestyle, we conducted this cross-sectional study. The aim of the present work was 1) to compare bone health and related variables (i.e., physical function, 25-hydroxy-vitamin D (250HD), parathyroid hormone (PTH) and BMD) between old adults living alone vs those in cohabitation; and 2) to investigate whether differences in lifestyle explain differences in bone health between the groups.
This was a secondary data analysis from baseline data of a previously published randomized, controlled trial, designed to examine the effect of post-exercise protein ingestion on the efficacy of strength training in old people [18].

\section{Methods}

Several papers have been published from this study and in the present paper we followed the methods of Geirsdottir et al. 2012 [19], Arnarson et al. 2013 [3], Arnarson et al. 2014 [20] and Ramel et al. $2015\left[{ }^{21}\right]$. The similarity found between the present paper and the above mentioned articles is restricted to the Methods chapter. The present paper contains novel scientific content with focus on living alone and bone health not presented previously.

\section{Subjects}

Participants ( $\mathrm{N}=236$, range 65-92 years) were recruited within the Capital Area in Iceland. Exclusion criteria were major orthopedic disease, pharmacological interventions known to affect muscle mass and low cognitive function according to Mini-Mental State Examination (MMSE) $\leq 19$ points) [12,22]. Subjects had also to be free from disorders that could significantly affect their body composition. Some of the participants had hypertension, hyperlipidemia or type 2 diabetes [23] although the enrolled subjects looked apparently healthy. The study received permission from the Icelandic National Bioethics Committee (VSNb2008060007/03-15) and has therefore been performed in accordance with the ethical standards as described by the Declaration of Helsinki 1964 and its later amendments. The subjects gave their written informed consent before participation in the study.

\section{Body composition and bone mineral density}

Body weight was measured in light underwear (Seca scale model no. 708, Seca, Hamburg, Germany) and height was measured with a calibrated stadiometer (model no. 206; Seca, Hamburg, Germany). Body mass index (BMI) was calculated as $\mathrm{kg} / \mathrm{m}^{2}$ and obesity defined as BMI $\geq 30 \mathrm{~kg} / \mathrm{m}^{2}$. Body composition (lean body mass $=\mathrm{LBM}$ ) and BMD (presented as z-scores for femur, lumbar and total) was assessed using dual energy x-ray absorptiometer (DXA, Hologic QDR-2000 plus ${ }^{\circledR}$, Hologic Inc., Waltham, MA, USA) at the Icelandic Heart Association, Kopavogur, Iceland.

\section{Physical function}

Six Minute Walk for Distance (6MWD)

The 6MWD (m) was conducted in a gym hall according to the guidelines from the American Thoracic Society [24]. 


\section{Grip strength}

Hand grip strength (lb.) was measured using a hydraulic hand dynamometer (Baseline ${ }^{\circledR}$ Baseline Evaluations Corporation). The maximal achieved grip strength from three attempts was recorded as the subject's grip strength.

\section{Questionnaires}

Questionnaires were used to receive information on socio-demographic characteristics, i.e., education (categorized as "high" vs. "low"), alcohol, current smoking, fish oil use (all categorized as "yes" and "no") and medication count. Participants' cohabitation status was retrospectively categorized into married/cohabitation and single/divorced/widowed.

\section{Leisure-time physical activity (LTPA)}

LTPA during the last year was estimated using a questionnaire [25] based on the Compendium of Physical Activities [26] and the Paffenberger's questionnaire [27]. It contains questions on the subjects' participation in sports, exercises or other physical activities. In statistical analysis LTPA is used as min/week.

\section{Biochemical measurements}

Participants were instructed to avoid strenuous exercise and alcohol consumption the day before the drawing of fasting blood samples at baseline and endpoint. The blood samples were centrifuged and the serum was stored at $-80^{\circ} \mathrm{C}$ for subsequent analysis at the University Hospital in Reykjavik, Iceland. Measurement of 250HD (nmol/L) was conducted by a chemiluminescence immunoassay using the DiaSorin LIAISON 250HD TOTAL assay (DiaSorin, Inc., Stillwater, Minnesota). Serum intact parathyroid hormone (PTH, pg/mL) was measured by chemiluminometric assay (Chiron Diagnostic corporation, USA).

\section{Statistical analysis}

Statistical analysis was conducted using SPSS for Windows version 22.0 (SPSS, Chicago, IL, USA). The Kolmogorov-Smirnov test was used to investigate the distribution of the data. In table 1 , data are shown as mean \pm standard deviation (SD). Comparison between groups, e.g., married/cohabitation vs. single/divorced/ widowed, was done using independent samples' t-test (normally distributed variables) or Mann-Whitney-U test (not normally distributed variables). Chi-square statistics were used to investigate differences in distribution of categorical variables between participants married/cohabitation and single/divorced/widowed.

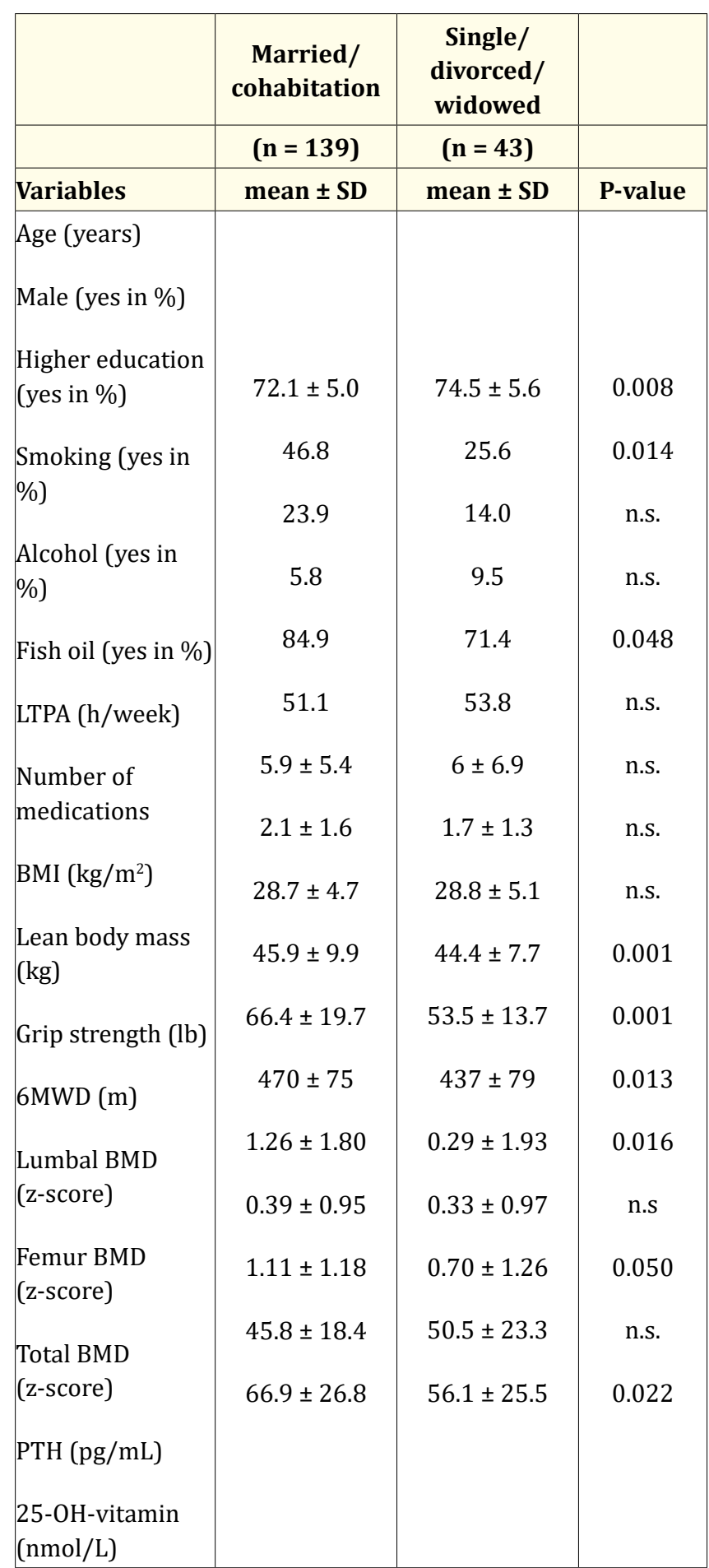

Table 1: Baseline characteristics of the participants $(\mathrm{N}=182)$.

Multivariate general linear models (GLM) were used to investigate differences in grip strength, 6MWD, BMD, 250HD and PTH be- 
Living Alone is Associated with Poorer Muscular Strength, Lower 25-hydroxy-vitamin D and Lower Bone Mineral Density in Icelandic Community Dwelling Old Adults

tween participants being married/in cohabitation vs participants being divorced/single/widowed. Results in table 2 are shown as parameter estimates using two different statistical models for each outcome: models 1 include intercept, age, gender and cohabitation status; models 2 additionally include BMI, fish oil intake, education and physical activity. Effect sizes $B$ give the estimated differences in the outcome variables between the two cohabitation groups. The level of significance was set at $\mathrm{P}<0.05$.

\begin{tabular}{|c|c|c|c|c|c|c|c|c|}
\hline \multirow[b]{3}{*}{ Parameter } & \multicolumn{4}{|c|}{ Model 1} & \multicolumn{4}{|c|}{ Model 2} \\
\hline & \multicolumn{4}{|c|}{ (corrected for age and gender) } & \multicolumn{4}{|c|}{$\begin{array}{l}\text { (additionally corrected for education, physical } \\
\text { activity, BMI and fish oil) }\end{array}$} \\
\hline & B & \multicolumn{2}{|c|}{$95 \% \mathrm{CI}$} & P-value & B & \multicolumn{2}{|c|}{$95 \% \mathrm{CI}$} & P-value \\
\hline \multicolumn{4}{|c|}{ Dependent Variable: grip strength (lb) } & & & & & \\
\hline Intercept & 88.855 & 58.738 & 118.972 & 0.001 & 79.820 & 41.284 & 118.357 & 0.001 \\
\hline Age (years) & -0.621 & -1.032 & -0.209 & 0.003 & -0.609 & -1.073 & -0.144 & 0.011 \\
\hline Male $^{1}$ & 33.531 & 29.493 & 37.569 & 0.001 & 34.290 & 30.034 & 38.545 & 0.001 \\
\hline Married/in cohabitation ${ }^{2}$ & 6.165 & 1.412 & 10.918 & 0.011 & 6.930 & 1.992 & 11.867 & 0.006 \\
\hline Basic education $^{3}$ & & & & & 2.061 & -2.510 & 6.632 & 0.373 \\
\hline LTPA (hours/week) & & & & & 0.160 & -0.230 & 0.550 & 0.418 \\
\hline BMI $\left(\mathrm{kg} / \mathrm{m}^{2}\right)$ & & & & & 0.199 & -0.249 & 0.648 & 0.380 \\
\hline Fish oil "no"4 & & & & & -2.341 & -6.568 & 1.885 & 0.274 \\
\hline \multicolumn{9}{|l|}{ Dependent Variable: 6MWD } \\
\hline Intercept & 937.308 & 752.755 & 1121.861 & 0.001 & 1010.123 & 804.060 & 1216.187 & 0.001 \\
\hline Age (years) & -6.892 & -9.415 & -4.370 & 0.001 & -5.967 & -8.452 & -3.482 & 0.001 \\
\hline Male $^{1}$ & 19.745 & -5.000 & 44.491 & 0.117 & 22.454 & -0.302 & 45.210 & 0.053 \\
\hline Married/in cohabitation ${ }^{2}$ & 26.556 & -2.570 & 55.682 & 0.074 & 22.727 & -3.677 & 49.131 & 0.091 \\
\hline Basic education $^{3}$ & & & & & -9.822 & -34.266 & 14.623 & 0.427 \\
\hline LTPA (hours/week) & & & & & 2.641 & 0.556 & 4.726 & 0.014 \\
\hline BMI $\left(\mathrm{kg} / \mathrm{m}^{2}\right)$ & & & & & -5.063 & -7.461 & -2.664 & 0.001 \\
\hline Fish oil "no"4 & & & & & 4.552 & -18.047 & 27.150 & 0.690 \\
\hline \multicolumn{9}{|c|}{ Dependent Variable: BMD lumbal (z-scores) } \\
\hline Intercept & -3.040 & -8.217 & 2.137 & 0.247 & -6.085 & -12.651 & 0.482 & 0.069 \\
\hline Age (years) & 0.041 & -0.029 & 0.112 & 0.249 & 0.070 & -0.010 & 0.149 & 0.084 \\
\hline Male $^{1}$ & 0.192 & -0.502 & 0.886 & 0.585 & 0.313 & -0.412 & 1.038 & 0.394 \\
\hline Married/in cohabitation ${ }^{2}$ & 1.195 & 0.378 & 2.012 & 0.005 & 1.354 & 0.512 & 2.195 & 0.002 \\
\hline Basic education $^{3}$ & & & & & 0.342 & -0.437 & 1.121 & 0.385 \\
\hline LTPA (hours/week) & & & & & 0.046 & -0.020 & 0.113 & 0.169 \\
\hline $\operatorname{BMI}\left(\mathrm{kg} / \mathrm{m}^{2}\right)$ & & & & & 0.007 & -0.069 & 0.083 & 0.857 \\
\hline Fish oil "no"4 & & & & & 0.246 & -0.474 & 0.966 & 0.500 \\
\hline \multicolumn{9}{|c|}{ Dependent Variable: femur (z-score) } \\
\hline Intercept & -1.598 & -4.313 & 1.118 & 0.246 & -3.555 & -7.021 & -0.089 & 0.045 \\
\hline Age (years) & 0.025 & -0.012 & 0.062 & 0.179 & 0.038 & -0.004 & 0.080 & 0.075 \\
\hline Male $^{1}$ & -0.405 & -0.769 & -0.040 & 0.030 & -0.355 & -0.737 & 0.028 & 0.069 \\
\hline
\end{tabular}


Living Alone is Associated with Poorer Muscular Strength, Lower 25-hydroxy-vitamin D and Lower Bone Mineral Density in Icelandic Community Dwelling Old Adults

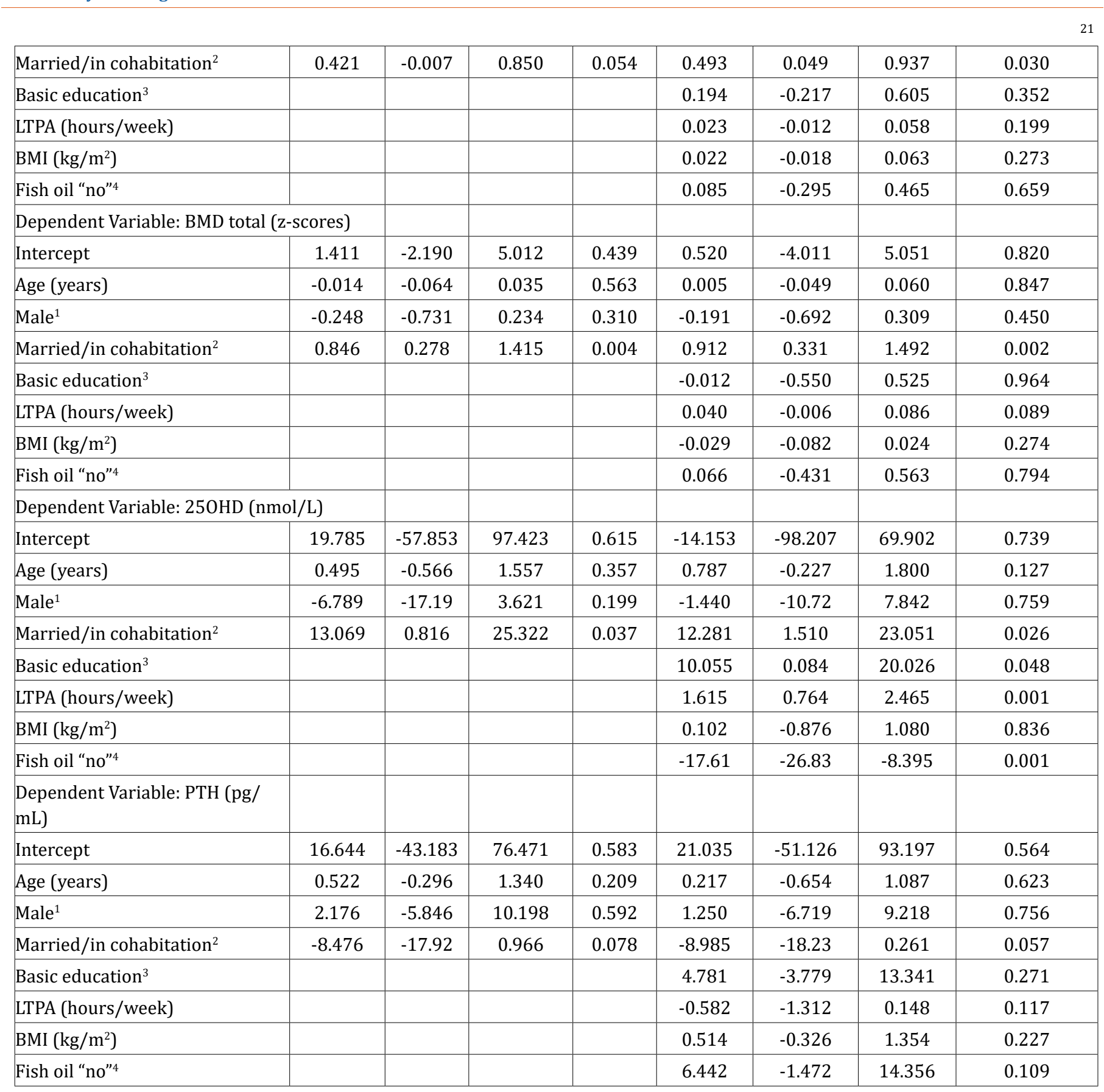

Table 2: Parameter estimates derived from multivariate GLM describing the differences between participants being married/in cohabitation $(n=139)$ vs participants being divorced/single/widowed $(n=43)$.

${ }^{1}$ as compared to female; ${ }^{2}$ as compared to single/divorced/widowed; ${ }^{3}$ as compared to higher education; ${ }^{4}$ as compared to fish oil "yes".

\section{Results}

Data were available for 182 participants, i.e., 106 women and 76 men. The characteristics of participants categorized by cohabita- tion status can be seen in table 1. Gender distribution between the cohabitation categories was uneven and women were overrepre- 
sented in the single/divorced/widowed category $(\mathrm{P}=0.014)$. Variables related to socioeconomics and lifestyle did not differ between the categories (with the exception of more frequent alcohol intake and younger age reported by married/in cohabitation), however measurements of physical strength, -function, BMD and 250HD were consistently more favorable in the married/in cohabitation category.

In order to further investigate these differences and to see whether confounding of age, gender distribution or other covariates explain findings from simple bivariate statistics in table 1, we conducted multivariate GLM. After correction for age and gender (Table 2, models 1), cohabitation status was no longer significantly related to 6MWD (but borderline), but otherwise, GLM largely confirmed the bivariate findings. Further correction for BMI, fish oil, LTPA and education (Table 2, models 2) did only marginally change effect sizes B and P values (in both directions). Although many of these covariates were by themselves significantly related to several of the outcome variables, they did not explain the differences between cohabitation categories.

\section{Discussion}

This cross-sectional study investigated the relationship between living alone and bone health (and related variables) and compared old adults living alone with those in cohabitation. We found that measurements of physical strength, -function, BMD and 250HD were significantly better in the married/in cohabitation category, however, these differences were not explained by different lifestyle of the two groups.

The differences in muscle strength and physical function, 250HD and BMD between the two cohabitation categories were considerable or around 0.5 SD. Similar results have been reported previously, i.e., individuals living alone have significantly lower BMD and greater odds of osteoporosis as well as of greater fracture risk than individuals living in cohabitation [7-10], although not all studies are in agreement [11]. As participants in the living alone category were significantly older and more often women, we used GLM to find out whether these differences persist after correction for these socio-economic confounders. It showed that social characteristics did not explain the observed differences in outcome variables between categories, although it should be mentioned that the difference in 6MWD decreased from $33 \mathrm{~m}$ (bivariate analysis) to $27 \mathrm{~m}$ (no longer significant) after correction for age and gender.
Mechanisms which could potentially explain such differences between social categories are not fully understood but may involve well described modifiable lifestyle factors [12-17]. We hypothesized that relevant lifestyle variables, i.e., LTPA, fish oil intake and BMI would explain differences in bone health related outcome variables between the cohabitation categories. For these lifestyle variables there is empirical evidence available linking them to bone health [28-30]. However, when comparing the groups in the bivariate analysis, there was not difference in lifestyle between them. Consequently, correction for lifestyle in the GLM did not alter the associations between cohabitation status and bone health related outcome variables.

Our study shows that cohabitation status matters regarding bone health related variables, however, it does not deliver any potential answer why participants living alone do worse compared to their counterparts in cohabitation. In that context it is important to consider residual confounding. Residual confounding describes the problem in epidemiology when a potential confounder is not or not fully accounted for due to measurement error or simply due to non consideration in the statistical analysis. Further, some of the observed differences between cohabitation categories, e.g., BMD, are the result of a live long interplay between environmental variables and genetic characteristics and thus a potentially important covariate, measured at one time point during lifetime, fails to show significant associations.

Other reasons for our findings could include misreporting which has been shown to be more in individuals in a lower social class leading to underestimation of the disparity when using selfreported information [31].

However, marriage and cohabitation have been shown to be closely related to health [32] and married people experience lower morbidity and mortality across diverse health threats as cancer, heart attacks and surgery [33]. Conversely, living alone is associated with increased risk for cardiovascular disease and all-cause mortality [34]. There are several reasons why cohabitation may exert a beneficial health impact. Partners spend time together during a wide variety of activities, such as eating, leisure, housekeeping, child-care and sleep [29]. Spouses also often share resources and investments such as joint finances or home-ownership and partnership serves as a source for intense support [29]. 


\section{Strength and limitations}

It is a strength of the present study that we measured and included many potential confounders with the purpose to explain differences between cohabitation categories. However, the present study was of cross-sectional nature and can thus not distinguish between cause and consequence for an observed association. Further, we did not have information on for how long participants have been in in cohabitation or living alone.

\section{Conclusion}

This cross-sectional study found that physical strength, -function, BMD and 250HD were significantly better in participants who were married or in cohabitation in comparison to participants living alone. Interestingly, these differences were not explained by different lifestyle of the two groups reported at old age. The observed differences might be a consequence of inequalities affecting outcomes during a longer time period.

\section{Conflict of Interest}

The authors declare no conflict of interest.

\section{Acknowledgement}

The study was funded by the research project Social inequalities in Ageing (SIA) -- health, care and institutional reforms in the Nordic welfare model funded by NordForsk (Project no.: 74637), the Icelandic Technology Development Fund (No 071323008), Research Fund of the University of Iceland, Landspitali University Hospital Research Fund and the Helga Jonsdottir and Sigurlidi Kristjansson Geriatric Research Fund.

\section{Bibliography}

1. Lee JJY., et al. "Ten-year absolute fracture risk and hip bone strength in Canadian women with systemic lupus Erythematosus". Journal of Rheumatology 39 (2012): 1378-1384.

2. Nelson HD., et al. "Screening for osteoporosis: an update for the U.S. preventive services task force". Annals of Internal Medicine 153 (2010): W. 43.

3. Compston J. "Osteoporosis: social and economic impact". $R a-$ diologic Clinics of North America 48 (2010): 477-482.

4. Mariconda M., et al. "Factors predicting mobility and the change in activities of daily living after hip fracture: a 1-year prospective cohort study". Journal of Orthopaedic Trauma 30 (2016): 71-77.

5. Travison T., et al. "Accounting for racial/ethnic variation in bone mineral content and density: the competing influences of socioeconomic factors, body composition, health and lifestyle, and circulating androgens and estrogens". Osteoporosis International 22 (2011): 2645-2654.

6. Navarro MC., et al. "Osteoporosis and metabolic syndrome according to socio-economic status, contribution of PTH, vitamin D and body weight: the Canarian osteoporosis poverty study (COPS)". Clinical Endocrinology 78 (2013): 681-686.

7. Kim SW., et al. "Association between household size, residential area, and osteoporosis: analysis of 2008 to 2011 Korea National Health and Nutrition Examination Survey". Korean Journal of Internal Medicine 31 (2016): 712-721.

8. Øyen J., et al. "Low-energy distal radius fractures in middleaged and elderly women-seasonal variations, prevalence of osteoporosis, and associates with fractures". Osteoporosis International 21 (2010): 1247-1255.

9. Vestergaard P., et al. "Socioeconomic aspects of fractures within universal public healthcare: a nationwide case-control study from Denmark". Scandinavian Journal of Public Health 34 (2006): 371-377.

10. Korpelainen R., et al. "Lifelong risk factors for osteoporosis and fractures in elderly women with low body mass index--a population-based study". Bone 39 (2006): 385-391.

11. Nabipour I., et al. "Socioeconomic status and bone health in community-dwelling older men: the CHAMP Study". Osteoporosis International 22 (2011): 1343-1353.

12. Wang S., et al. "Social and behavior factors related to aged Chinese women with osteoporosis". Gynecology and Endocrinology 24 (2008): 538-545.

13. Kumar A., et al. "Impact of dietary intake, education, and physical activity on bone mineral density among north Indian women". Journal of Bone and Mineral Metabolism 28 (2010): 192-201.

14. Shin CS., et al. "Prevalence and risk factors of osteoporosis in Korea: a community-based cohort study with lumbar spine 
and hip bone mineral density". Bone 47 (2010): 378-387.

15. Hsieh C-H., et al. "Factors influencing osteoporosis preventive behaviours: testing a path model". Journal of Advanced Nursing 62 (2008): 336-345.

16. Brennan SL., et al. "Association between socioeconomic status and bone mineral density in adults: a systematic review". Osteoporosis International 22 (2011): 517-527.

17. Castro-Lionard K., et al. "Use of calcium supplements, vitamin D supplements and specific osteoporosis drugs among French women aged 75-85 years: patterns of use and associated factors". Drugs Aging 30 (2013): 1029-1038.

18. Arnarson A., et al. "Changes in body composition and use of blood cholesterol lowering drugs predict changes in blood lipids during 12 weeks of resistance exercise training in old adults". Aging Clinical and Experimental Research 26 (2014): 287-292.

19. Geirsdottir OG., et al. "Physical function predicts improvement in quality of life in elderly Icelanders after 12 weeks of resistance exercise". The Journal of Nutrition, Health and Aging 16 (2012): 62-66.

20. Arnarson A., et al. "Changes in body composition and use of blood cholesterol lowering drugs predict changes in blood lipids during 12 weeks of resistance exercise training in old adults". Aging Clinical and Experimental Research 26(2014):287-292.

21. Ramel A., et al. "C-Reactive Protein and Resistance Exercise in Community Dwelling Old Adults". The Journal of Nutrition, Health and Aging 19 (2015): 792-796.

22. Folstein MF., et al. "A practical method for grading the cognitive state of patients for the clinician". Journal of Psychiatry Research 12 (1975): 189-198.

23. Geirsdottir OG., et al. "Effect of 12-Week Resistance Exercise Program on Body Composition, Muscle Strength, Physical Function, and Glucose Metabolism in Healthy, Insulin-Resistant, and Diabetic Elderly Icelanders". The Journals of Gerontology, Series A: Biological Sciences 67 (2012): 1259-1265.

24. "ATS Statement. Guidelines for the Six-Minute Walk Test". American Journal of Respiratory and Critical Care Medicine 166
(2002): 111-117.

25. Martínez-González MA., et al. "Prevalence of physical activity during leisure time in the European Union". Medicine and Science in Sports and Exercise 33 (2001): 1142-1146.

26. Ainsworth BE., et al. "Compendium of physical activities: an update of activity codes and MET intensities". Medicine and Science in Sports and Exercise 2 (2000): s498-s504.

27. Paffenbarger RS., et al. "Paffenbarger physical activity questionnaire". American Journals of Epidemiology 108 (1978): 161-175.

28. Eymundsdottir H., et al. "Serum 25-hydroxy vitamin D, physical activity and cognitive function among older adults". Journal of Aging Research 7 (2018): 143-148.

29. Geirsdottir OG., et al. "Effect of 12-Week Resistance Exercise Program on Body Composition, Muscle Strength, Physical Function, and Glucose Metabolism in Healthy, Insulin-Resistant, and Diabetic Elderly Icelanders". Journals of Gerontology: Series A 67(2012):1259-1265.

30. Ramel A., et al. "Vitamin D deficiency and nutritional status in elderly hospitalized subjects in Iceland". Public Health Nutrition 12.7 (2009): 1001-1005.

31. Ljungvall A., et al. "Misreporting and misclassification: implications for socioeconomic disparities in body-mass index and obesity". The European Journal of Health Economics 16 (2015): 5-20.

32. Robles TF., et al. "Marital quality and health: a meta-analytic review". Psychology Bulletin 140 (2014): 140-187.

33. Kiecolt-Glaser JK and Newton TL. "Marriage and health: his and hers". Psychology Bulletin 127 (2001): 472-503.

34. Hawkley LC and Cacioppo JT. "Loneliness matters: a theoretical and empirical review of consequences and mechanisms". Annals of Behavioral Medicine's 40 (2010): 218-227.

\section{Volume 5 Issue 10 October 2021 (C) All rights are reserved by Ramel A., et al.}

